\title{
Sensitivity Pattern of Salmonella serotypes in Northern India
}

\author{
Vikas Gautam, Naveen Kumar Gupta, \\ Uma Chaudhary and D. R. Arora
}

\author{
Department of Microbiology, PGIMS, Rohtak, India
}

\begin{abstract}
Background. Enteric fever continues to bea major public health problem, especially in the developing countries of the tropics. We determined the incidence of Salmonella bloodstream infections and their antimicrobial resistance patterns from May to August in the years 1997-2001 in Haryana, a large state of India. The minimum inhibitory concentration (MIC) was also determined for 60 isolates of $S$. typhi to various commonly used antimicrobial agents. Material and Methods. Blood cultures of 6,956 patients (PUO/septicemia) were processed by standard procedures and the Salmonella spp. isolates were identified with specific antisera and with standard biochemical tests. Antimicrobial susceptibilities were determined by Stokes disc diffusion method. The MIC of 60 randomly isolated strains of S. typhi was determined by agar dilution method using Mueller Hinton Agar medium. Results. Isolation rates of Salmonella spp. increased in 2000 and 2001. Multidrug resistance (MDR) in S. typhi had increased while in $S$. paratyphi it had decreased markedly. Ninety per cent chloramphenicol sensitivity was seen in S.typhi by MIC method. There was a decrease in the susceptibility to ciprofloxacin of S. typhi with MIC showing an upward trend. All S.typhi tested were sensitive to third generation cephalosporins and aminoglycosides with MIC well below the breakpoint. Discussion. Our study indicates that MDR in $S$. typhi is on the rise in our area. There is also re-emergence of chloramphenicol sensitivity. Rising MIC values of ciprofloxacin may lead to prolonged treatment, delayed recovery or pose treatment failure. Thus, sensitivity pattern of causative organism must be sought before instituting appropriate therapy to prevent further emergence of drug resistance.

Key Words: Salmonella, multidrug resistance.
\end{abstract}

Enteric fever is a worldwide problem and widely prevalent in the developing countries of the tropics. An estimated 600,000 deaths from enteric fever occur annually throughout the world [1]. Salmonella typhi and $S$. paratyphi A are the predominant types of Salmonella responsible for enteric fever in India, particularly in the summer [2]. In India, S. typhi drug resistance has been reported since 1960; followed by the first outbreak of multidrug resistant $S$. typhi (MDRST) in Calicut [3,4]. Since then MDRST has appeared throughout the world, especially in South

Received on 02 August 2002; revised 27 November 2002.

Address for correspondence: Dr. Naveen Kumar Gupta, Senior Resident, Microbiology. D-87, Ashok Vihar, Phase-I, Delhi, India-110052. Phone: (91)-11-7420625.

E-mail:micronaveen@hotmail.com

The Brazilian Journal of Infectious Diseases 2002;6(6):281-287 (C) 2002 by The Brazilian Journal of Infectious Diseases and Contexto Publishing. All rights reserved. $1413-8670$
America, the Indian subcontinent, Africa and Southeast Asia [5-7]. Drug resistance is of considerable importance to microbiologists and is posing a major therapeutic problem for the public and for public health authorities. Resistance to commonly used antibiotics, such as chloramphenicol, ampicillin, and co-trimoxazole has been reported from different parts of India in the last two decades [8]. In the recent past, fluoroquinolones and cephalosporins have gained importance for the treatment of enteric infections. Considerable variation in antibiotic resistance patterns among salmonellae to various antibiotics has been reported from different parts of India. Hence we studied the incidence of Salmonella bloodstream infections and their antimicrobial resistance patterns from May to August, 1997 to 2001. Minimum inhibitory concentrations (MIC) were also determined by agar dilution method for 60 randomly isolated (May to August, 2001) strains of S. typhi. 


\section{Material and Methods}

Blood cultures of 6,956 patients (PUO/septicemia) attended at the Postgraduate Institute of Medical Sciences, Rohtak, Haryana and outpatient departments from May to August, 1997 to 2001 were processed by standard procedures [9]. Duplicate isolates from the same patient were excluded from the study.

\section{$\underline{\text { Microbiological methods }}$}

All isolates of Salmonella spp. from blood were identified with specific antisera(Central Research Institute, Kasauli, India) and with standard biochemical tests [9]. Antimicrobial susceptibilities were determined at the time of isolation by the Stokes disc diffusion method, with discs

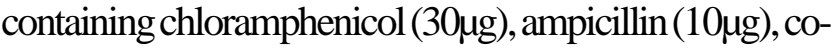
trimoxazole $(25 \mu \mathrm{g})$, gentamicin $(10 \mu \mathrm{g})$, amikacin $(10 \mu \mathrm{g})$,

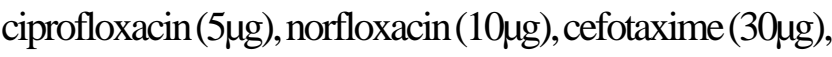

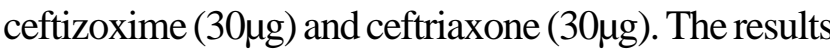
were interpreted using standard methods [10]. MIC of 60 isolates of S. typhiwas determined by the agar dilution method using Mueller Hinton Agar medium (Hi Media, India) with an inoculum of $10^{5}$ organisms $/ \mathrm{ml}$, followed by 24 hours incubation and interpreted using a standard protocol [10]. The control strain was NCTC 10418 Escherichia coli. Each isolated Salmonella was tested against 12 dilutions of 8 antibiotics viz. ampicillin, chloramphenicol, gentamicin, amikacin, ciprofloxacin, cefotaxime, ceftizoxime and ceftriaxone.

\section{Results}

A total of 533 isolates of Salmonella spp. were obtained from 6956 blood cultures. S. typhi was the predominant serotype, followed by S. paratyphi A and S. typhimurium. There were recent increases in Salmonella bloodstream infections in 2000 (7.7\%) and 2001 (9.3\%), when compared to previous years (Table 1).

The antimicrobial susceptibility pattern of S. typhi against various antibiotics tested was ampicillin (21\% to $45 \%$ ), chloramphenicol ( $41 \%$ to $52 \%$ ), cotrimoxazole (15\% to $74 \%$ ), gentamicin ( $57 \%$ to $76 \%$ ), norfloxacin (34\% to $83 \%$ ), amikacin ( $76 \%$ to $87 \%$ ), ciprofloxacin (62\% to $89 \%$ ), cefotaxime ( $74 \%$ to $88 \%$ ), ceftizoxime (89\% to $95 \%$ ), ceftriaxone (87\% to $90 \%$ ) (Table 2). While ampicillin, cotrimoxazole and norfloxacin showed a downward trend, chloramphenicol showed an upward trend in the sensitivity pattern. All the newer drugs, eg. fluoroqinolones and cephalosporins were more effective, however some resistance problems, ranging from 5\% to $25 \%$ have been encountered recently.

The antimicrobial sensitivity pattern of S.paratyphi against the various antibiotics (from 1997 to 2001) is shown in Table 3. There was decreased sensitivity pattern against chloramphenicol, ampicillin, cotrimoxazole and norfloxacin (12\% to 38\%) while the better sensitivity pattern was shown by aminoglycosides, fluoroquinolones and cephalosporins (70\% to 87\%) in the year 2001 .

Multidrug resistance (MDR) i.e. resistance to three or more antibiotics in S.typhiand S.paratyphi $\mathrm{A}$ is as shown in Table 4. Overall MDR (from 1997 to 2001) in S. typhi was found in $242(55.5 \%)$ out of 436 isolates and in 34 (36.2\%) out of 94 isolates in S. paratyphi A. MDR in $S$. typhi showed an upward trend while S. paratyphi A showed a downward trend. According to the antibiotic resistance (ACCo) 38 of 436 isolates of S. typhiand 10 of 94 isolates of S. paratyphi were multidrug resistant. Out of these 48 MDR isolates, 36 were even resistant to amikacin, cefotaxime, ciprofloxacin, ceftizoxime and ceftriaxone by disc diffusion method.

The MIC of 60 isolates of S. typhi was determined; $100 \%$ of these salmonellae were found to be resistant to ampicillin (MIC $=128 \mu \mathrm{g} / \mathrm{ml}$ ), $90 \%$ of the strains were sensitive to chloramphenicol (breakpoint MIC $=32 \mu \mathrm{g} / \mathrm{ml}$ ) while only $63 \%$ of the strains were sensitive to ciprofloxacin (break point MIC $=4 \mu \mathrm{g} / \mathrm{ml}$ ). S. typhi strains were found to be sensitive to gentamicin ( $0.015 \mu \mathrm{g}$ to $2 \mu \mathrm{g} / \mathrm{ml})$, amikacin $(0.06 \mu \mathrm{g}$ to $2 \mu \mathrm{g} / \mathrm{ml})$, cefotaxime $(0.03 \mu \mathrm{g}$ to $0.5 \mu \mathrm{g} / \mathrm{ml})$, ceftizoxime ( $0.06 \mu \mathrm{g}$ to $1 \mu \mathrm{g} / \mathrm{ml})$, ceftriaxone $(0.06 \mu \mathrm{g}$ to $0.5 \mu \mathrm{g} /$ $\mathrm{ml}$ ) below their MIC break point (Table 5).

\section{Discussion}

Enteric fever is a major public health problem in our country. Isolation of Salmonella spp. occurs throughout the year in Haryana. This means that 
Table 1. Prevalence of Salmonella spp. from May to August in 1997 to 2001

\begin{tabular}{lcccccc}
\hline & $\mathbf{1 9 9 7}(\boldsymbol{\%})$ & $\mathbf{1 9 9 8}(\boldsymbol{\%})$ & $\mathbf{1 9 9 9}(\boldsymbol{\%})$ & $\mathbf{2 0 0 0}(\boldsymbol{\%})$ & $\mathbf{2 0 0 1}(\boldsymbol{\%})$ & Total(\%) \\
\hline Total no.of blood & & & & & & \\
samples received & 1167 & 1101 & 1162 & 1743 & 1783 & 6956 \\
S. typhi & $56(4.7)$ & $38(3.5)$ & $42(3.6)$ & $134(7.7)$ & $166(9.3)$ & $436(6.3)$ \\
S. paratyphi A & $16(1.4)$ & $14(1.3)$ & $12(1.0)$ & $5(0.3)$ & $47(2.6)$ & $94(1.4)$ \\
S. typhimurium & $03(0.3)$ & 0 & 0 & 0 & 00 & $3(0.04)$ \\
Total no. of salmonellae & $65(5.6)$ & $52(47)$ & $54(4.6)$ & $139(8.0)$ & $213(12)$ & $533(7.7)$ \\
\hline
\end{tabular}

Table 2. Sensitivity (in \%) of Salmonella typhi to various antibiotics by the disc diffusion method

\begin{tabular}{lcccccc}
\hline Antibiotics/Year & $\mathbf{1 9 9 7}$ & $\mathbf{1 9 9 8}$ & $\mathbf{1 9 9 9}$ & $\mathbf{2 0 0 0}$ & $\mathbf{2 0 0 1}$ & Overall \\
\hline Chloramphenicol & 41 & 41 & 43 & 48 & 52 & 45 \\
Ampicillin & 27 & 37 & 31 & 45 & 21 & 32 \\
Gentamicin & 65 & 68 & 57 & 76 & 68 & 67 \\
Co-trimoxazole & 74 & 47 & 40 & 13 & 15 & 38 \\
Norfloxacin & 83 & 79 & 60 & 65 & 34 & 64 \\
Amikacin & 85 & 85 & 76 & 87 & 87 & 84 \\
Ciprofloxacin & 89 & 76 & 62 & 87 & 81 & 79 \\
Cefotaxime & 74 & 76 & 74 & 82 & 88 & 79 \\
Ceftizoxime & - & - & - & 89 & 95 & 92 \\
Ceftriaxone & - & - & - & 87 & 90 & 88 \\
\hline
\end{tabular}

Table 3. Sensitivity (in \%) of Salmonella paratyphi to various antibiotics by the disc diffusion method

\begin{tabular}{lccccc}
\hline Antibiotics/Year & $\mathbf{1 9 9 7}$ & $\mathbf{1 9 9 8}$ & $\mathbf{1 9 9 9}$ & $\mathbf{2 0 0 0}$ & $\mathbf{2 0 0 1}$ \\
\hline Chloramphenicol & 25.0 & 35.7 & 50.0 & 80.0 & 72.8 \\
Ampicillin & 50.0 & 57.1 & 33.3 & 40.0 & 14.9 \\
Gentamicin & 62.5 & 57.1 & 75.0 & 80.0 & 70.2 \\
Co-trimoxazole & 50.0 & 50.0 & 33.3 & 40.0 & 38.3 \\
Norfloxacin & 87.5 & 78.6 & 41.7 & 60.0 & 14.9 \\
Amikacin & 75.0 & 92.9 & 91.7 & 100.0 & 83.0 \\
Ciprofloxacin & 93.8 & 64.2 & 83.3 & 100.0 & 83.0 \\
Cefotaxime & 62.5 & 100.0 & 83.3 & 100.0 & 85.1 \\
Ceftizoxime & - & - & - & 100.0 & 87.2 \\
Ceftriaxone & - & - & - & 100.0 & 85.1 \\
\hline
\end{tabular}


Table 4. Multidrug resistance among Salmonella spp.

\begin{tabular}{lccccccc}
\hline Years & \multicolumn{3}{c}{ S. typhi } & & \multicolumn{3}{c}{ S. paratyphi } \\
\cline { 2 - 3 } & Total & MDR ( \%) & ACCo(\%) & & Total & MDR (\%) & ACCo(\%) \\
\hline 1997 & 56 & $30(53.6)$ & $2(3.6)$ & & 16 & $11(68.8)$ & $0(0)$ \\
1998 & 38 & $19(50.0)$ & $6(15.8)$ & & 14 & $05(35.7)$ & $2(14.3)$ \\
1999 & 42 & $24(57.0)$ & $7(16.7)$ & & 12 & $05(41.7)$ & $2(16.7)$ \\
2000 & 134 & $63(47.0)$ & $10(7.5)$ & & 5 & $00(20.0)$ & $0(0)$ \\
2001 & 166 & $106(63.9)$ & $13(7.8)$ & & 47 & $13(27.7)$ & $5(10.6)$ \\
Total & 436 & $242(55.5)$ & $38(8.7)$ & & 94 & $34(36.2)$ & $10(10.6)$ \\
\hline
\end{tabular}

MDR = Multidrug resistance

$\mathrm{ACCo}=$ Antibiotic resistance (ampicillin, chloramphenicol, co-trimoxazole).

Table 5. MIC values of $S$. typhi to various antimicrobial agents

\begin{tabular}{|c|c|c|c|c|c|c|c|c|}
\hline $\begin{array}{c}\text { Drug } \\
(\mu \mathrm{g} / \mathrm{ml})\end{array}$ & $\begin{array}{c}\text { Ampi } \\
(\geq 32) *\end{array}$ & $\begin{array}{l}\text { Chlor } \\
(\geq 32)\end{array}$ & $\begin{array}{c}\text { Genta } \\
(\geq 8)\end{array}$ & $\begin{array}{c}\text { Amika } \\
(\geq 32)\end{array}$ & $\begin{array}{c}\text { Cipro } \\
(\geq 4)\end{array}$ & $\begin{array}{c}\text { Cefotax } \\
(\geq 64)\end{array}$ & $\begin{array}{l}\text { Ceftiz } \\
(\geq 32)\end{array}$ & $\begin{array}{c}\text { Ceftriax } \\
(\geq 64)\end{array}$ \\
\hline$\geq 128$ & 60 & 04 & $-* *$ & - & - & - & - & - \\
\hline 64 & 0 & 0 & - & - & - & 0 & 0 & 0 \\
\hline 32 & 0 & 02 & - & - & - & 0 & 0 & 0 \\
\hline 16 & 0 & 12 & 0 & 0 & 0 & 0 & 0 & 0 \\
\hline 08 & 0 & 02 & 0 & 0 & 12 & 0 & 0 & 0 \\
\hline 04 & 0 & 16 & 0 & 0 & 10 & 0 & 0 & 0 \\
\hline 02 & 0 & 14 & 14 & 02 & 04 & 0 & 0 & 0 \\
\hline 01 & 0 & 0 & 42 & 56 & 0 & 0 & 02 & 0 \\
\hline 0.5 & 0 & 04 & 0 & 0 & 0 & 02 & 0 & 22 \\
\hline 0.25 & 0 & 0 & 0 & 0 & 20 & 10 & 0 & 34 \\
\hline 0.12 & 0 & 0 & 0 & 0 & 0 & 02 & 0 & 0 \\
\hline 0.06 & 0 & 06 & 02 & 02 & 06 & 12 & 58 & 04 \\
\hline 0.03 & - & - & 0 & 0 & 0 & 34 & 0 & 0 \\
\hline 0.015 & - & - & 02 & 0 & 0 & - & - & - \\
\hline 0.007 & - & - & 0 & 0 & 08 & - & - & - \\
\hline
\end{tabular}

Numbers in each row indicate the number of strains with that MIC value against each antimicrobial agent.

Ampi, ampicillin; Chlor, chloramphenicol; Genta, gentamicin; Amika, amikacin; Cipro, ciprofloxacin; Cefotax, cefotaxime; Ceftizox, ceftizoxime; Ceftriax, ceftriaxone.

* = breakpoint MIC.

** $=$ dilution not made of that particular antibiotic.

drinking water conditions and sanitation have not improved or a large number of carriers are present in the society. Isolation rates of Salmonella spp. have increased in recent years (2000-2001), particularly in the summer months. Proper sanitation, public health education and vaccination are long term preventive measures that would improve this situation.
Chloramphenicol has been the mainstay of treatment for enteric fever, while ampicillin/co-trimoxazole are other cost-effective and well-tried primary drugs of choice. Drug resistance to chloramphenicol in S. typhi first emerged in the United Kingdom(UK) in the 1950s and subsequently in Greece and Israel followed by the epidemics of MDR Salmonella in Mexico, India and 
other regions [11]. Though the resistance to chloramphenicol increased steadily in India from 1960 onwards the ACCo showed a downwards trend from $15.8 \%$ to $7.8 \%$ from 1998 to 2001 (Table 1). This indicates a reemergence of chloramphenicol sensitivity in S. typhi (Table 2), as reported previously $[12,13]$. MIC determination by the tube dilution method also showed that $90 \%$ of $S$. typhi strains were chloramphenicol sensitive in year 2001 while ampicillin resistance was $100 \%(\mathrm{MIC}=128 \mu \mathrm{g} / \mathrm{ml})$.

An interesting feature that was observed in various other studies conducted worldwide as well as in our study was the lack of correlation between the results of disc diffusion and MIC methods [14-18]. While MIC was determined for only 60 isolates in 2001, we tested a large number of isolates with the disc diffusion method. Similar to our chloramphenicol sensitivity results, Jevanand et al. [14] found that $29.6 \%$ of the strains were sensitive to chloramphenicol by the disc diffusion method while $100 \%$ sensitivity was observed with MIC methodology. All the strains were found to be resistant to ampicillin by MIC methodology by Jevanand et al as determined in our study [14]. We observed an increased resistance to ampicillin with MIC methodology $(=128 \mu \mathrm{g} /$ $\mathrm{ml}$ in all the strains) when compared to the disc diffusion method (79\% of the strains resistant in 2001). In a similar manner, more strains were considered resistant to drugs by MIC than with disc diffusion in another study [15]. These variations could also be due to fewer isolates being tested with MIC and through random selection of the isolates. This latter hypothesis is supported by the fact that when we used both methods on the 60 strains isolated in 2001 there was almost $90 \%$ correlation between the two methods. A study was conducted in Britain where each of 12 laboratories in different parts of Britain supplied approximately 80 consecutive bacterial isolates to Department of Bacteriology, St. Mary's Hospital, London. Not all laboratories provided sensitivity reports for every antimicrobial substance for which MIC determinations were made. There were sometimes considerable discrepancies between the two sets of data. Consequently, as stated by Barrett et al., Stokes results may not always correlate well with those determined by MIC or breakpoint [16].
MDRST is a major therapeutic concern for physicians in developing countries. Contributory factors may be drug overuse, misuse and inappropriate prescribing practices by physicians along with intrinsic microbiological plasmid-mediated factors. In the years 1982-89 the rate of MDRST in India was below 15\%, but it increased to $50 \%$ in 1990 , to more than $70 \%$ in 1992 [19] and then to $90 \%$ in Bangalore in 1994 [20]. In our region, MDRST varied from 38.5\% in 1990 [21] to $27.11 \%$ in 1995 [22]. We found MDR in $S$. typhito increase from $53.6 \%$ to $63.9 \%$, while in $S$. paratyphi A it dropped from $68.8 \%$ to $27.7 \%$ from 1997 to 2001 (Table 4).

In a search for improved treatments for enteric fever and MDRST in particular, attention has been focused on fluoroquinolone compounds and broad-spectrum cephalosporins because of their excellent properties. These highly active drugs reduce the duration of treatment from the traditional 14 days that is necessary with chloramphenicol. Short treatment regimens and reduced periods of hospitalization have obvious financial benefits, particularly in developing countries. They are also more likely to ensure compliance [23]. In our study there was $88 \%$ sensitivity to ciprofloxacin while sensitivities to third generation cephalosporins cefotaxime, ceftizoxime and ceftriaxone were $81 \%$, $90 \%$ and $95 \%$, respectively, by the disc diffusion method in 2001. However, the MIC of S. typhi to ciprofloxacin has shown an upward trend with $20 \%$ strains with $\mathrm{MIC}=8 \mu \mathrm{g} / \mathrm{ml}$ and $16.6 \%$ strains with $\mathrm{MIC}$ $=4 \mu \mathrm{g} / \mathrm{ml}$ (breakpoint MIC inoculum $=4 \mu \mathrm{g} / \mathrm{ml}$ ). It is important to remember that the ability of bacteria to develop resistance is partly related to the concentrations of the drugs to which they are exposed. It is therefore recommended that therapeutic blood levels of antibiotics should be four times higher than MIC. This has been observed for quinolones by Cullmann et al., but this aspect of pharmacodynamics needs to be investigated [24,25]. However, all S. typhi tested were sensitive to third generation cephalosporins (MIC range 0.03 to $1 \mu \mathrm{g} / \mathrm{ml}$ ) and aminoglycosides (gentamicin and amikacin), with MIC well below the breakpoint. However, parenterally applied broad spectrum cephalosporins are inferior to fluoroquinolones and it 
is unlikely that oral drugs of the same class would be any better [26].

The use of fluoroquinolone antibiotics in children is contraindicated because of dose-dependent druginduced damage to the articular cartilage of weight bearing joints. However, fluoroquinolones have now been safely used in typhoid and other types of systemic salmonellosis, as well as for other childhood lifethreatening infections such as MDR pseudomonas and drug resistant shigellosis [27,28]. In our study, 5-10\% drug resistance was observed to third generation cephalosporins by $S$. typhi with the disc diffusion method, though all the strains tested had MIC values considerably below the breakpoint MIC. Similar differences have been reported by other researchers [17].

Thus our study indicates that MDR in S. typhi is on the rise in our area. On the other hand there has been a reemergence of chloramphenicol sensitivity. Rising MIC values of ciprofloxacin may lead to prolonged treatment, delayed recovery or post treatment failure [29]. Thus the sensitivity pattern of causative organism must be studied before instituting appropriate therapy to prevent further emergence of drug resistance.

\section{Acknowledgements}

Thanks are due to Dr. Nitin M. Nagarkar, MS, DNB, Reader, ENT, Government Medical College and Hospital, Chandigarh for rendering his help. We are also thankful to Prof. Narottam Sharma, MA(Eng) MEd MCTE PGDTE (Hyd) Retd from the Regional Institute of English Chandigarh, U.T. for proof reading the manuscript.

\section{References}

1. Eddman R., Levine M.M. Summary of an international workshop on typhoid fever. Infect Dis 1986;8:329-49.

2. Gupta B., Kumar U., Khurana S. Multidrug resistant Salmonella Typhi in Ludhiana (Punjab). Indian J Pathol Microbiol 1993;36(1):5-7.

3. Agarwal S.C. Chloramphenicol resistance of Salmonella species in India, 1956-61. Bull Wld Hlth Orgn 1962;17: 331-5.

4. Panicker C.K.J., Vimla K.N. Transferable chloramphenicol resistance in Salmonella Typhi. Nature 1972;239:109-10.
5. Butler T., Linch N., Arnold K., Pollack M. Chloramphenicol resistant typhoid fever in Vietnam associated with $\mathrm{R}$ factor. Lancet 1973;ii:983-5.

6. El-Sherbini A. An outbreak of typhoid fever resistant to chloramphenicol and other drugs in Gharbeya Govemorate. Egypt J Trop Pediatr 1992;38:331-4.

7. Mourad A.S., Matwally M., Nour Ei Deen A., et al. Multiple drug-resistant Salmonella Typhi. Clin Infect Dis 1993; $17: 135-6$

8. Sanghavi S.K., Mane M.P., Niphadkar K.B. Multidrug resistance in Salmonella serotypes. Indian J Med Microbiol 1999;17(2):88-90.

9. Old D.C. Salmonella. In: Collee J.G., Marmion B.P., Fraser A.G., Simmons A. eds. Practical Medical Microbiology, 14th edn. London: Churchill Livingstone, 1996.

10. National Committee for Clinical Laboratory Standards (NCCLS). Performance Standards for Antimicrobial Susceptibility Testing - Sixth edition; Approved Standards M2-A6. 1997, Wayne, Pennsylvania.

11. Mirza S.H., Beeching N.J., Hart C.A. Multi-drug resistant typhoid: a global problem. J Med Microbiol 1996;44:317-9.

12. Bhattacharya S.S., Das U. Occurrence of Salmonella Typhi infection in Rourkela, Orissa. Indian J Med Res 2000; $111: 75-6$.

13. Sood S., Kapil A., Das B., et al. Re-emergence of Chloramphenicol-sensitive Salmonella Typhi. Lancet 1999;353:1241.

14. Jevanand H.R., Ragavan P.U.M., Gunapathi R.S. Study of R-factors among multidrug resistant Salmonella Typhi. Indian J Med Microbiol 1997;15(1):37-9.

15. Hemalatha R., Vijayalakshmi P., Gyaneshwari, et al. Multidrug resistant Salmonella Typhi in Hyderabad. Indian J Med Microbiol 1999; 17(1):39-41.

16. Barrett S.P., Savage M.A., Rebec M.P., et al. Antibiotic sensitivity of bacteria associated with communityacquired urinary tract infection in Britain. J Antimicrob Chemother 1999;44:359-65.

17. Bhat K.G., Andrade A.T., Karadesai S.G., et al. Antimicrobial susceptibility of Salmonella Typhi to quinolones and cephalosporins. Indian J Med Res 1998; $107: 247-51$.

18. Bhat K.G., Metgud S.C., Patil C.S. Fluoroquinolone resistance in Gram negative bacteria. Indian Pract 1992; $45(1): 35-8$.

19. Pillai P.K., Prakash K. Current status of drug resistance and phage types of Salmonella Typhi in India. Indian J Med Res 1993;97:154-8.

20. Ratish K.C., Chandrashekar M.R., Nagesh C.N. Multidrug resistant Salmonella Typhi in Bangalore. South Indian J Med Sci 1994;48:85-8.

21. Sabherwal U., Chaudhary U. Multidrug resistance $S$. Typhi in Haryana 1989-1990. Indian J Med Res 1992;95:12-3. 
22. Sikka R., Arora D.R., Saini S. Multi drug resistant Salmonella Typhi in Rohtak. Indian J Med Microbiol 1996; 14:53-4.

23. Smith M.D., Duong N.M., Hoa N.T.T., et al. Comparison of ofloxacin and ceftriaxone for short-course treatment of enteric fever. Antimicrob Agents Chemother 1994;38:1716-20.

24. Jesudason M.V., Malathy B., JacobJohn T. Trend of increasing levels of minimum inhibitory concentration of ciprofloxacin to Salmonella Typhi. Indian J Med Res 1996; $103: 247-9$.

25. Cullmann W., Stieglitz M., Baars B., et al. Comparative evaluation of recently developed quinolone compounds - with a note on the frequency of resistant mutants. Chemoptherapy 1985;31:19-28.

26. Vinh H.A., Wain J., Hanh V.T.N., et al. Two or three days of ofloxacin treatment for uncomplicated resistant typhoid fever in children. Antimicrob Agents Chemother 1996;40(4):958-61.

27. Hien T.T., Bethell D.B., Hoa N.T., et al. Short course of ofloxacin for treatment of multidrug-resistant typhoid. Clin Infect Dis 1995;20:917-23.

28. Schoad U.B. Toxicity of quinolones in paediatric patients. Adv Antimicrob Antineoplast Chemother 1992; 11:259-65.

29. Threlfall E.J., Ward L.R., Skinner J.A., et al. Ciprofloxacinresistant Salmonella Typhi and treatment failure. Lancet 1999;353:1590-1. 